Gynäkologische Endokrinologie 2009 · 7:190-190 DOI 10.1007/s10304-009-0324-7

Online publiziert: 16. August 2009

(c) Springer Medizin Verlag 2009

\section{Redaktion}

T. Steck, Passau

\author{
M. Kousehlar · G. Allgayer - C. Daufratshofer - M. Haseitl · R. Felberbaum \\ KinderWunschKempten, Zentrum für Reproduktionsmedizin, \\ Klinikum Kempten - Oberallgäu, Kempten
}

\title{
Ei mit doppeltem Dotter
}

\section{Das Phänomen der „binovular zona pellucida“}

Das Phänomen zweier Eizellen innerhalb einer einzigen Zona pellucida, gewonnen aus einem einzigen Follikel, tritt selten auf. Es wurde erstmals von Papadaki et al. 1978 [1] beschrieben. Zur Entstehung des Phänomens existieren 2 Theorien: Entweder ist es das Ergebnis einer abnormalen meiotischen Teilung mit dem Ergebnis eines monströs vergrößerten Polkörperchens [2] oder aber die Folge der Tatsache, dass 2 Eizellen sehr nahe beieinander lagen, sodass es während der Follikelbildung nicht zur Trennung kam [3]. In dem hier vorliegenden Fall konnten 2 solcher ,binovularen Zonae" bei einer einzigen Patientin (40-jährige o-Gravida, $\mathrm{COH}$ zur IVF mit ICSI bei andrologisch bedingter Ehesterilität) gefunden werden ( $\bullet$ Abb. 1).

\section{Korrespondenzadresse}

Prof. Dr. R. Felberbaum

KinderWunschKempten,

Zentrum für Reproduktionsmedizin,

Klinikum Kempten - Oberallgäu

Robert-Wexler-Str. 50, 87439 Kempten

ricardo.felberbaum@klinikum-kempten.de

\section{Literatur}

1. Papadaki L (1978) Binovular follicles in the adult ovary. Fertil Steril 29:342-350

2. Ben-Rafael Z, Mastroianni L, Kopf GS (1987) In-vitro-fertilization and cleavage of a single egg from a binovular follicle containing two individual eggs surrounded by a single zona pellucida. Fertil Steril 47:707-709

3. Zeilmaker GH, Alberda AT, VanGent I (1983) Fertilization and cleavage of oocytes from a binovular human ovarian follicle: a possible cause of dizygotic twinning and chimerism. Fertil Steril 40:841843

Interessenkonflikt. Keine Angaben.
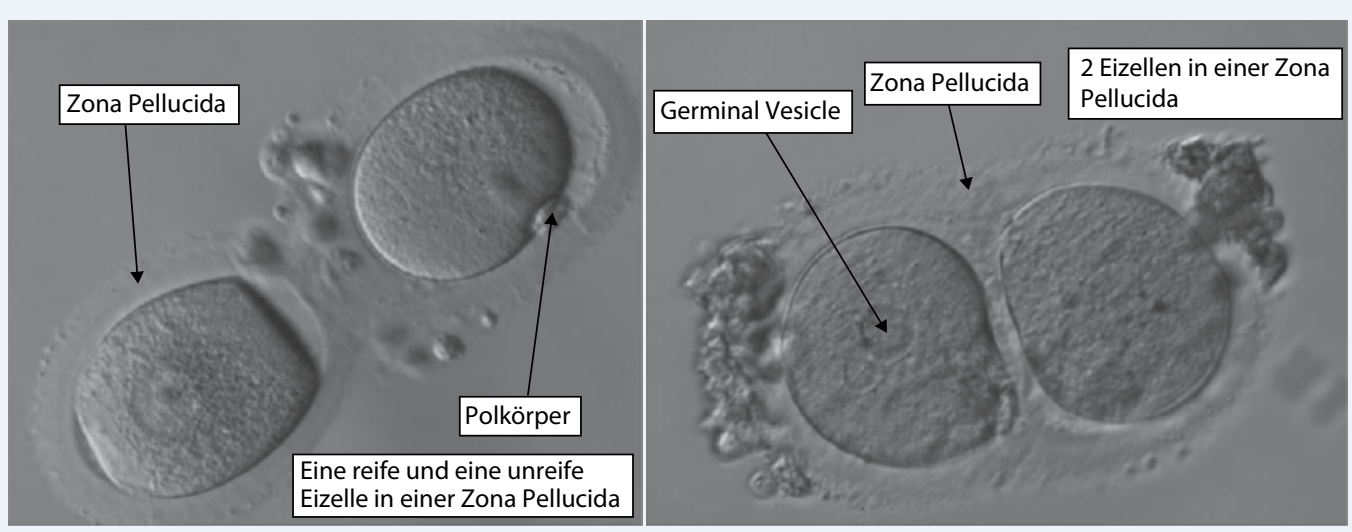